\title{
Neurociência e o ensino de programação: Uma revisão sistemática da literatura
}

\author{
Marina O. Queiroz, Ayla Débora Dantas S. Rebouças \\ Universidade Federal da Paraíba (UFPB) \\ Departamento de Ciências Exatas (DCX) - Rio Tinto, PB - Brazil \\ \{marina.oliveira, ayla\}@dce.ufpb.br
}

\begin{abstract}
With high failure rates in programming disciplines at universities, there is a need to find a way to tackle this problem. Possible solutions to this difficulty can be found in the techniques discovered by neuroscience that can help in the teaching-learning process. This article presents a Systematic Literature Review (SLR) whose main objective is to seek papers in the field of neuroscience that present techniques that may enable the development of some useful skills for programming disciplines, such as logical reasoning.
\end{abstract}

Resumo. Com altos índices de reprovação em disciplinas de programação nas universidades, surge a necessidade de encontrar uma forma de atacar esta problemática. Possíveis soluções para esta dificuldade podem ser encontradas nas técnicas descobertas pela neurociência que podem ajudar no processo de ensino-aprendizagem. O presente artigo apresenta uma Revisão Sistemática da Literatura (RSL) que tem como principal objetivo buscar trabalhos na área da neurociência que apresentem técnicas que possam permitir o desenvolvimento de algumas habilidades úteis para as disciplinas de programação, como, por exemplo, o raciocínio lógico.

\section{Introdução}

Os altos índices de reprovação nas disciplinas de programação têm sido um problema constante nos cursos das áreas tecnológicas. Segundo Raabe e Silva (2005), essas disciplinas exigem o desenvolvimento de estratégias de resolução de problemas com base lógico-matemática, que para muitos são altamente abstratas e distantes do cotidiano. Contudo, o desenvolvimento da habilidade de raciocínio é um problema que pode ser atacado através de técnicas de aprendizagem baseadas nas descobertas da neurociência, que segundo Santos e Vasconcelos (2014) é a ciência que estuda o sistema nervoso e seu funcionamento.

Devido ao déficit no desenvolvimento de tais habilidades, foi feita uma investigação através de uma revisão sistemática da literatura para buscar descobertas da neurociência na área de ensino e aprendizagem, que possam ser aplicadas no ensino das disciplinas de programação, proporcionando aos alunos o desenvolvimento de habilidades como o raciocínio lógico. Sternberg (2005) destaca que a inteligência humana é altamente maleável e que pode ser moldada e incrementada por meio de vários tipos de intervenções.

Como afirmam Santos e Vasconcelos (2014), a interlocução entre a neurociência e a educação demonstra que as estratégias pedagógicas que respeitam como o cérebro 
VII Congresso Brasileiro de Informática na Educação (CBIE 2018)

Anais do XXIX Simpósio Brasileiro de Informática na Educação (SBIE 2018)

funciona, têm maior possibilidade de serem mais eficientes, pois as descobertas da neurociência oferecem uma abordagem mais científica no processo de ensinoaprendizagem, apoiada na compreensão dos processos cognitivos envolvidos.

O objetivo deste trabalho é identificar descobertas da neurociência na área de ensino e aprendizagem que podem ser utilizadas no ensino das disciplinas de programação ou para o desenvolvimento de habilidades na área, como o raciocínio lógico.

Este trabalho foi dividido em seis etapas: i) Na primeira etapa foi definida a questão de pesquisa que norteou a revisão sistemática; ii) $\mathrm{Na}$ segunda etapa foram definidas as fontes de busca dos trabalhos; iii) Em uma terceira etapa foram selecionados os trabalhos com base na definição das chaves de busca e através da leitura do título e do resumo, considerando os critérios de inclusão e exclusão definidos; iv) Na quarta etapa foram extraídos os dados de interesse para a pesquisa; v) $\mathrm{Na}$ quinta etapa foram sintetizados e estudados os dados para responder à questão de pesquisa definida na primeira etapa; vi) Na sexta etapa, foram descritos os resultados encontrados ao longo do trabalho.

Este trabalho está organizado conforme descrito a seguir: A Seção 2 apresenta a fundamentação teórica utilizada neste trabalho; na Seção 3 é definido o protocolo de pesquisa utilizado para a realização dessa revisão sistemática; na Seção 4 são apresentados os resultados da pesquisa; na Seção 5 é encontrada a análise dos dados obtidos desta pesquisa; e, por fim, a Seção 6 apresenta as considerações finais sobre esse trabalho e propostas de trabalhos futuros.

\section{Fundamentação Teórica}

A neurociência estuda os neurônios e suas moléculas constituintes, os órgãos do sistema nervoso e suas funções específicas. Conforme afirmam Cosenza e Guerra (2011), os neurocientistas têm como objetivo principal decifrar os comandos do cérebro e todas as suas funções, o que é muito importante pois o cérebro controla todas as nossas ações, como, por exemplo, nossa memória e nossas habilidades motoras. Um outro aspecto importante a destacar é que a neurociência é uma ciência interdisciplinar, o que implica dizer que ela tem relação com diversas áreas como: biologia, química, medicina, matemática, linguística, engenharia, física e ciência da computação.

O cérebro controla todas as nossas ações, como por exemplo, as emoções, o apetite, a respiração, o sono, e tudo que fazemos. O cérebro possui hemisférios distintos, onde cada um deles é dedicado a um conjunto de funções. As principais áreas do cérebro são as seguintes: lobo frontal, lobo parietal, lobo temporal, lobo occipital, cerebelo e tronco cerebral, como mostra a Figura 1. 
VII Congresso Brasileiro de Informática na Educação (CBIE 2018)

Anais do XXIX Simpósio Brasileiro de Informática na Educação (SBIE 2018)

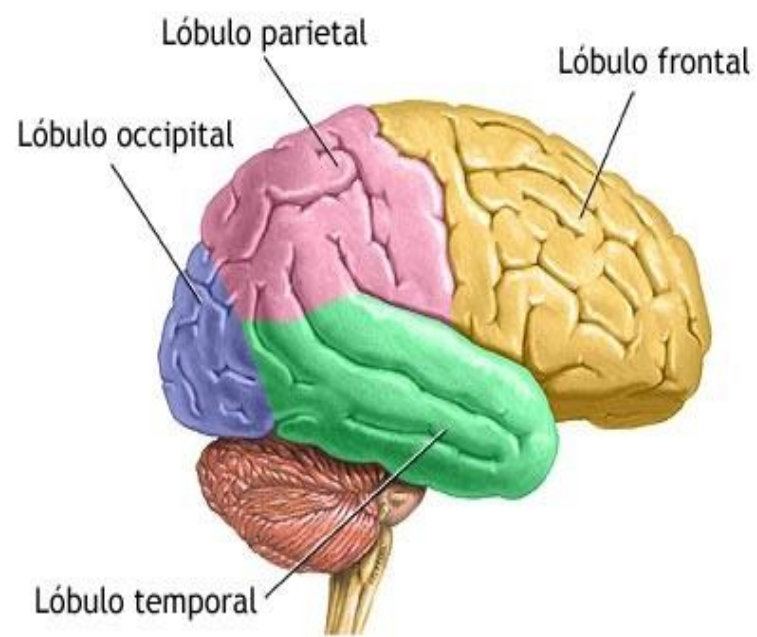

Principais áreas do cérebro. ${ }^{1}$

Figura 1.

O cérebro é composto por cerca de 86 bilhões de neurônios, cuja principal função é transportar mensagens por meio de processos eletroquímicos. O neurônio converte o sinal elétrico em sinal químico, com o objetivo de passar a informação para outro neurônio e esse neurônio converte a mensagem de volta por um impulso elétrico para executar o processo. Os neurônios processam toda a informação que flui no interior do sistema nervoso central.

\subsection{Neurociência e Aprendizagem}

A neurociência descreve a estrutura e funcionamento do sistema nervoso, enquanto a educação cria condições que promovem o desenvolvimento de competências [Souza, 2016]. Como afirmam também Coch e Ansari (2009), os professores atuam como agentes nas mudanças cerebrais que levam à aprendizagem.

Para que o cérebro "aprenda", é utilizada a memória de trabalho que se localiza no lobo frontal e concentra todas as informações e estímulos que estão sendo recebidos do meio. Também é usada a memória de longo prazo que está localizada por toda a região do córtex cerebral. Para que o cérebro armazene as informações, a memória de trabalho filtra os estímulos que estão sendo recebidos do meio externo e busca na memória de longo prazo informações que estão relacionadas com a informação que ele absorveu e transporta essas informações para a memória de trabalho. Através do cruzamento das informações novas com as antigas acontece o "raciocínio lógico". Quanto mais esse processo se repete, as informações novas são transformadas e transportadas para a memória de longo prazo [Cosenza e Guerra, 2011] [Kandel, Schartz e Jessel, 1997] [Lent, 2004].

Segundo Bastos, Adamatti e Carvalho (2015), a neurociência cognitiva afirma que a aprendizagem e a memória estão fortemente relacionadas, sendo a memória a base orgânica para que ocorra a aprendizagem. Memorizar não é o mesmo que aprender, mas para que ocorra aprendizagem, é preciso que as informações fiquem armazenadas na nossa memória.

\footnotetext{
1 Disponível em <http://www.anatomiadocorpo.com/sistema-nervoso/cerebro/>. Acesso em $15 / 10 / 2017$
} 
VII Congresso Brasileiro de Informática na Educação (CBIE 2018)

Anais do XXIX Simpósio Brasileiro de Informática na Educação (SBIE 2018)

\subsection{Neurociência e Ensino de Programação}

O aprendizado de programação exige dos alunos o pensamento lógico, pensamento este que segundo Tassano e Duarte (2015), leva a um processo de resolução de problemas baseado em etapas com resultados que podem servir para a decisão/resolução de etapas subsequentes. Todo ser possui o pensamento lógico, porém, este, precisa ser aperfeiçoado para o processo de resolução de problemas.

Para que ocorra o pensamento lógico, algumas áreas do cérebro precisam ser acessadas. Quando o pensamento lógico é pouco "aperfeiçoado" o aluno tende a acessar mais áreas do cérebro do que necessário, pois este toma vários caminhos para conseguir chegar ao resultado. Já o aluno que tem um pensamento lógico mais "aperfeiçoado" tende a acessar apenas as áreas do cérebro necessárias para a resolução daquele problema, como comprova o experimento feito por Bastos, Adamatti e Carvalho (2015).

Segundo Tassano e Duarte (2015), os conceitos subsunçores ${ }^{2}$, aliados ao que nos traz o estudo da neurociência cognitiva, nos levam a crer que se treinado o desenvolvimento do pensamento lógico, pode-se aproveitar o cérebro mais do que se imagina. Por exemplo, acredita-se que exercícios de lógica como um "aquecimento" para o cérebro podem ser essenciais para um melhor rendimento nas disciplinas de programação.

\section{Protocolo de Pesquisa}

Este artigo apresenta os resultados de uma revisão sistemática da literatura analisando os artigos encontrados de acordo com um protocolo de pesquisa pré-estabelecido. Segundo Kitchenham (2007), uma revisão sistemática da literatura é um meio de identificar, avaliar e interpretar todas as pesquisas disponíveis relevantes para uma determinada questão de pesquisa, área de tópico ou fenômeno de interesse de forma não enviesada e até certo ponto repetível.

Inicialmente pensou-se em fazer um Mapping Study, que é um tipo de revisão sistemática, porém, pelo fato de um Mapping Study ser um estudo quantitativo e o objetivo desta pesquisa é identificar quais são as evidências e não a quantidade de evidências existentes de técnicas da neurociência para apoiar o ensino de programação, um mapeamento sistemático mais abrangente ficou como proposta de trabalho futuro. Kitchenham (2007) afirma que os estudos de mapeamento sistemático são projetados para fornecer uma ampla visão geral de uma área de pesquisa, para estabelecer se existem evidências de pesquisa sobre um tópico e fornecer uma indicação da quantidade de evidências. Como o presente trabalho tem um caráter mais exploratório, de identificar técnicas existentes, sem a pretensão de identificar todas, mas considerando que o processo de identificar é sistemático, acreditou-se que ele fica melhor caracterizado apenas como revisão sistemática da literatura, mas mapeamentos que considerem a área de neurociência e desenvolvimento de raciocínio lógico de forma mais ampla são futuras pesquisas vislumbradas após este trabalho.

\subsection{Questões de Pesquisa}

2 Ausubel define este conhecimento prévio como "conceito subsunçor" ou simplesmente "subsunçor". Os subsunçores são estruturas de conhecimento específicos que podem ser mais ou menos abrangentes de acordo com a freqüência com que ocorre aprendizagem significativa em conjunto com um dado subsunçor. 
VII Congresso Brasileiro de Informática na Educação (CBIE 2018)

Anais do XXIX Simpósio Brasileiro de Informática na Educação (SBIE 2018)

Esta revisão sistemática da literatura tem como tema central de pesquisa as técnicas da neurociência na área de ensino e aprendizagem e que podem ser úteis no ensino das disciplinas de programação, proporcionando aos alunos o desenvolvimento de habilidades como o raciocínio lógico. $\mathrm{O}$ tema central da revisão sistemática norteará a questão de pesquisa abordada neste artigo. Foi definida a seguinte questão de pesquisa:

- QP1: Quais as principais técnicas sugeridas por pesquisas da neurociência que podem apoiar o ensino-aprendizagem de programação?

\subsection{Fontes de Busca}

As fontes de busca utilizadas para esse trabalho foram encontradas a partir do portal de periódicos da CAPES (Comissão de Aperfeiçoamento de Pessoal do Nível Superior). Foram utilizadas as seguintes fontes: ACM (Association for Computing Machinery), arxiv.org (Cornell UniversityLibrary), IEEE Xplore Digital Library e SciELO (Scientific Eletronic Library Online).

Para conduzir a pesquisa foram definidas chaves de busca específicas. Foram aplicadas em todas as fontes de busca três combinações diferentes de chaves, entretanto, para determinadas fontes de busca, os resultados eram relevantes apenas com uma ou duas chaves. Tais chaves foram: i) neurosciences AND education AND programming; ii) neurosciences AND learning; iii) neuroscienses AND education. Essas mesmas chaves de busca foram utilizadas também em português, porém, não foi encontrado dessa forma nenhum trabalho além dos que já tinham sido encontrados. Nessa pesquisa também foram utilizadas diversas outras chaves de busca, entretanto, as citadas acima foram as únicas que tiveram resultados relevantes para a pesquisa.

\subsection{Critérios de Inclusão e Exclusão}

Para a seleção dos artigos foram estabelecidos alguns critérios de inclusão e exclusão. Após a definição desses critérios foi feita a análise dos artigos em duas etapas: i) Leitura do resumo, observando se atendia aos critérios de inclusão ou exclusão e incluindo-os em uma pré-seleção; ii) Leitura completa dos trabalhos pré-selecionados para extração dos dados dos trabalhos que foram incluídos. A seleção, análise e extração dos dados foi feita por uma das autoras sob a supervisão da segunda autora deste trabalho de forma a sanar dúvidas durante o processo e auxiliar na estruturação da pesquisa e dos seus resultados.

Tabela 1. Critérios de inclusão e exclusão

\begin{tabular}{|l|l|}
\multicolumn{1}{|c|}{ Critérios de Inclusão (CI) } & Critérios de Exclusão (CE) \\
\hline $\begin{array}{l}\text { CI01 - Foram selecionados trabalhos } \\
\text { que abordam o tema neurociência e } \\
\text { ensino de programação; }\end{array}$ & $\begin{array}{l}\text { CE01 - Não foram incluídos trabalhos que } \\
\text { abordavam apenas o tema ensino de } \\
\text { programação; }\end{array}$ \\
\hline $\begin{array}{l}\text { CI02 - Foram selecionados trabalhos } \\
\text { que abordam o tema neurociência e } \\
\text { educação; }\end{array}$ & $\begin{array}{l}\text { CE02 - Não foram incluídos trabalhos que } \\
\text { abordavam apenas o tema neurociência; }\end{array}$ \\
\hline
\end{tabular}


VII Congresso Brasileiro de Informática na Educação (CBIE 2018)

Anais do XXIX Simpósio Brasileiro de Informática na Educação (SBIE 2018)

CI03 - Foram selecionados trabalhos que abordam o tema neurociência, ensino e aprendizagem.
CE03 - Não foram incluídos trabalhos que abordavam apenas o tema educação ou ensino e aprendizagem.

\section{Resultados}

Após as pesquisas utilizando as chaves de busca citadas anteriormente, foram encontrados um total de 685 trabalhos, onde, desse total puderam ser extraídos 8 trabalhos utilizando os critérios de inclusão e exclusão definidos na seção anterior deste trabalho. É importante destacar que as pesquisas foram feitas entre os dias 08 de Agosto e 20 de Setembro de 2017.

Tabela 2. Resultados encontrados

\begin{tabular}{|c|c|c|c|c|c|}
\hline $\begin{array}{l}\text { Fontes de } \\
\text { Busca }\end{array}$ & $\begin{array}{c}\text { Chaves de } \\
\text { Busca }\end{array}$ & $\begin{array}{c}\text { Trabalhos } \\
\text { Encontrados }\end{array}$ & $\begin{array}{c}\text { Trabalhos } \\
\text { pré- } \\
\text { selecionados }\end{array}$ & $\begin{array}{l}\text { Trabalhos } \\
\text { incluídos }\end{array}$ & $\begin{array}{c}\text { Trabalhos } \\
\text { incluídos (\%) } \\
\text { em relação ao } \\
\text { total de } \\
\text { trabalhos } \\
\text { encontrados }\end{array}$ \\
\hline $\mathrm{ACM}$ & $\begin{array}{l}\text { Neurosciences } \\
\text { AND } \\
\text { education } \\
\text { AND } \\
\text { programming }\end{array}$ & 14 & 4 & 2 & $14.28 \%$ \\
\hline $\mathrm{ACM}$ & $\begin{array}{l}\text { Neurosciences } \\
\text { AND learning }\end{array}$ & 122 & 1 & 1 & $0,81 \%$ \\
\hline Arxiv.org & $\begin{array}{l}\text { Neurosciences } \\
\text { AND learning }\end{array}$ & 223 & 9 & 1 & $0,44 \%$ \\
\hline IEEE & $\begin{array}{c}\text { Neurosciences } \\
\text { AND } \\
\text { education } \\
\text { AND } \\
\text { programming }\end{array}$ & 41 & 2 & 1 & $2.43 \%$ \\
\hline Scielo & $\begin{array}{c}\text { Neurosciences } \\
\text { AND } \\
\text { education }\end{array}$ & 19 & 7 & 3 & $15.78 \%$ \\
\hline- & - & Total: 685 & Total: 24 & Total: 8 & Total: $1.16 \%$ \\
\hline
\end{tabular}

Após a análise dos trabalhos pré-selecionados, alguns foram excluídos da revisão pois abordavam apenas a importância do uso das técnicas descobertas pela neurociência no ensino, mas não identificavam quais técnicas poderiam ser utilizadas no processo de ensino e aprendizagem.

\section{Análise de Resultados}


VII Congresso Brasileiro de Informática na Educação (CBIE 2018)

Anais do XXIX Simpósio Brasileiro de Informática na Educação (SBIE 2018)

Após a revisão dos artigos, foram extraídos alguns dados que respondem a questão de pesquisa que fundamenta este trabalho. A QP1 faz o seguinte questionamento: Quais as principais técnicas sugeridas por pesquisas da neurociência que podem apoiar o ensinoaprendizagem de programação?

A Tabela 3 apresenta as pesquisas da neurociência que podem apoiar o ensinoaprendizagem de programação que foram encontradas durante a revisão. Nem todas as pesquisas abaixo citadas são exclusivamente voltadas para o ensino de programação, porém todas as técnicas abaixo citadas têm como objetivo principal desenvolver o raciocínio lógico dos alunos, que é o alicerce da programação.

Tabela 3. Principais técnicas sugeridas por pesquisas da neurociência

\begin{tabular}{|c|c|c|}
\hline Código & Técnica sugerida & Fonte que cita a técnica \\
\hline T01 & Brain Wave Gaming & [Kaul 2006] \\
\hline T02 & $\begin{array}{c}\text { Brain-Based Teaching } \\
\text { Methods }\end{array}$ & $\begin{array}{l}\text { [Sabitzer, Pasterk e Elsenbaumer } \\
\text { 2013] }\end{array}$ \\
\hline T03 & Brain Jam & [Pollock, Murray e Yeager 2017] \\
\hline T04 & Pprimming & [Redish 2014] \\
\hline T05 & Theory based on p-prims & [Redish 2004] \\
\hline T06 & Brain-based Programming & [Sabitzer $e$ Pasterk 2014] \\
\hline T07 & $\begin{array}{c}\text { Técnica baseada na temática } \\
\text { Freiriana }\end{array}$ & [Freitas, Motta e Mello-Carpes 2015] \\
\hline T08 & $\begin{array}{c}\text { Técnica baseada em atividades } \\
\text { Lúdicas }\end{array}$ & [Fernandes et al. 2015] \\
\hline
\end{tabular}

As técnicas citadas na Tabela 3 podem auxiliar os professores no processo de ensino e desenvolver nos estudantes habilidades como as de raciocínio lógico. Porém, observou-se que existem poucos trabalhos de pesquisa que sugerem métodos que podem apoiar o processo de ensino-aprendizagem usando técnicas descobertas pela neurociência.

\subsection{Técnicas sugeridas}

Abaixo estão descritas as técnicas encontradas contendo o código de cada trabalho em que a técnica de ensino se encontra.

T01 - Brain Wave Gaming: O método fornece um ambiente para exercitar o cérebro. É um tipo de jogo que permite aos alunos aprender através do aumento da capacidade intelectual. Esse método de exercitar permite que os alunos monitorem sua capacidade de animar objetos usando suas ondas cerebrais, através de um capacete que capta ondas cerebrais. Os alunos se adaptam a novas formas de aprendizagem, a fim de desenvolver novas formas de pensar e resolver problemas.

T02 - Brain-Based Teaching: É um modo de ensinar baseado no método COOL (Cooperative Open Learning), usando o método de programação em pares e a 
VII Congresso Brasileiro de Informática na Educação (CBIE 2018)

Anais do XXIX Simpósio Brasileiro de Informática na Educação (SBIE 2018)

metodologia de aprender ensinando, a fim de fazer com que os alunos desenvolvam melhor os conteúdos de programação.

T03 - Brain Jam: Esse método se baseia em jogos lúdicos em que o aluno deve primeiro fixar o conteúdo de forma lúdica e depois associar esse conteúdo ao real conteúdo da disciplina. A abordagem brain jam é interdisciplinar, interativa e centrada no problema e no design.

T04 - Pprimming: Esse método consiste em dar "10 minutos" para os alunos responderem questionários "regulares" e "difíceis" uma vez por semana. Os questionários são diretos e os problemas são solucionáveis e de resposta curta ou múltipla escolha, mas estão configurados para levar a uma resposta errada se os estudantes recorrerem ao pensamento de uma etapa e ignorarem o mecanismo pprimming (um recurso cognitivo correspondente a uma declaração básica sobre o funcionamento do mundo físico que um usuário considerava óbvio e irredutível). Muitas vezes, uma pergunta parecerá uma que os alunos já tiveram visto como uma pergunta da lição de casa ou em recitação mas com uma mudança na situação que leva a uma mudança na resposta. Essa mudança de situação faz com os alunos ignorem o mecanismo de pprimming e não sigam a intuição para responder a pergunta. Este tipo de mecanismo é utilizado para fazer os alunos repensarem em suas respostas.

T05 - Theory based on p-prims (phenomenological primitives): Esse método consiste em fazer com que os alunos exercitem o mecanismo de ppriming, onde o professor cria perguntas em que para responder eles devem seguir a intuição, assim melhorando a maneira do aluno pensar e desenvolver melhor a resposta antes de responder uma pergunta.

T06 - Brain-based Programming: O método consiste na realização de tarefas. Os alunos escolhem entre diferentes tipos de tarefas e temas de acordo com seu conhecimento. As aulas de 90 minutos são divididas em 3 unidades de aprendizagem (perguntas, descobrir e programação de pares), onde eles seguem seu próprio ritmo de aprendizagem.

T07 - Técnica baseada na temática Freiriana: O processo de IT (investigação temática), apregoado por Freire, está relacionado à visualização das imagens construídas no processo de codificação, com hipóteses de teóricos que enfatizam que a combinação de informações linguística e visual, apreendidas no processo de investigação, fornece duplo suporte para a aprendizagem e a construção de conhecimento. Estimula-se assim, por meio da IT, uma aprendizagem focada no contexto do aprendente visto que dessa forma seus sentimentos e emoções são mobilizados, o que favorece seu processo de aprendizagem.

T08 - Técnica baseada em atividades Lúdicas: O objetivo deste método é desenvolver algumas habilidades como as de: promover o desenvolvimento de habilidades do pensamento matemático e da resolução de problemas em alunos com indicativos de DA (Dificuldade de Aprendizagem) em cálculo, promover o desenvolvimento de habilidades cognitivas em geral usando o corpo e vivências psicomotoras e promover uma integração sensorial ao facilitar uma aprendizagem corporal - motora/psicomotora através de atividades lúdicas.

\section{Considerações Finais e Trabalhos Futuros}


VII Congresso Brasileiro de Informática na Educação (CBIE 2018)

Anais do XXIX Simpósio Brasileiro de Informática na Educação (SBIE 2018)

As técnicas encontradas nesta revisão sistemática da literatura foram apresentadas com o objetivo de investigar em futuros estudos primários como essas técnicas podem melhorar os resultados de aprendizagem dos alunos principalmente nas disciplinas de programação. Nem todos os trabalhos dessa RSL são totalmente voltados para o ensino de programação. Porém, há alguns trabalhos voltados para melhorar o desempenho dos alunos no modo de resolver problemas e desenvolver algumas habilidades que são extremamente úteis para o ensino de programação, sendo interessante pesquisar no futuro o uso destas técnicas no ensino de programação em si.

Após esse trabalho de pesquisa foi possível notar que as pesquisas em torno dos processos de ensino-aprendizagem baseados na neurociência ainda são muito escassas. Porém mostram-se bastante úteis pesquisas nessa área, pois o cérebro humano interfere totalmente no processo de ensino e aprendizagem, onde, através das técnicas descobertas por estudos neurocientíficos podemos modificar nos alunos sua percepção, o modo de pensar e resolver problemas, levando a um melhor desempenho escolar e acadêmico.

Devido a escassez de trabalhos na área não foi possível fazer análises estatísticas sobre os trabalhos encontrados, sendo um interessante trabalho futuro algum mapeamento sistemático ou revisão sistemática da literatura que identifique trabalhos com técnicas da neurociência voltadas ao desenvolvimento do raciocínio lógico em si, utilizando chaves de busca mais específicas. Após essa análise inicial dos métodos de ensino baseados na neurociência, um outro trabalho futuro também vislumbrado é realizar estudos mais aprofundados sobre a aplicação e funcionamento desses métodos em turmas de disciplinas de programação.

\section{Referências}

Bastos, N; Adamatti, D; e Carvalho, F. (2015). Ensino de Lógica de Programação no Ensino Médio e suas implicações na Neurociências. In: Anais do XXVI Simpósio Brasileiro de Informática na Educação - SBIE.

Coch, D. e Ansari, D. (2009). Thinking about mechanisms is crucial to connecting neuroscience and education. Cortex, 45, 546-547.

Cosenza, R. e Guerra, L. (2011). Neurociência e educação: como o cérebro aprende. Porto Alegre: Artmed.

Fernandes, C. (2015). et al. Possibilidades de aprendizagem: reflexões sobre neurociência do aprendizado, motricidade e dificuldades de aprendizagem em cálculo em escolares entre sete e 12 anos. Ciência \& Educação (Bauru), v. 21, n. 2.

Freitas, D; Motta, C. e Mello-Carpes, P. (2015). As bases neurobiológicas da aprendizagem no contexto da investigação temática freiriana.Trab. educ. saúde, p. 109-122.

Kaul, P. (2006). Neurological gaming environments. In:ACM SIGGRAPH 2006 Educators program. ACM, p. 2.

Kandel, E; Schartz e Jessel, T. (1997). Fundamentos da neurociência e do comportamento. Rio de Janeiro: Prentice-Hall do Brasil.

Kitchenham, B. (2007).”Guidelines for performing Systematic Literature Reviews in Software Engineering". 
VII Congresso Brasileiro de Informática na Educação (CBIE 2018)

Anais do XXIX Simpósio Brasileiro de Informática na Educação (SBIE 2018)

Lent, R. (2004). Cem bilhões de neurônios: conceitos fundamentais de neurociência. Athenue. São Paulo.

Mafra, S. e Travassos, G. (2006). Estudos Primários e Secundários apoiando a busca por Evidência em Engenharia de Software. Relatório Técnico, RT-ES, v. 687, n. 06,

Pinker, S. (1998). Como a mente funciona. São Paulo: Companhia das Letras.

Pollock, I; Murray, J. e Yeager, B. (2017). Brain jam: STEAM learning through neuroscience-themed game development. In: Proceedings of the Second International Conference on Game Jams, Hackathons, and Game Creation Events. ACM, p. 15-21.

Raabe, A. e Silva, J. (2005). Um Ambiente para Atendimento as Dificuldades de Aprendizagem de Algoritmos. In: XXV Congresso da Sociedade Brasileira de Computação. São Leopoldo/RS.

Redish, E. (2014). Oersted Lecture 2013: How should we think about how our students think?.

Redish, E. (2004). A theoretical framework for physics education research: Modeling student thinking. arXiv preprint physics/0411149, 2004.

Sabitzer, B; Pasterk, S. e Elsenbaumer, S. (2013). Brain-based teaching in computer science: neurodidactical proposals for effective teaching. In:Proceedings of the 13th Koli Calling International Conference on Computing Education Research. ACM, p. 197-198.

Sabitzer, B. e Pasterk, S. (2014). Brain-based programming continued: Effective teaching in programming courses. In:Frontiers in Education Conference (FIE), 2014 IEEE. IEEE, p. 1-6.

Santos, M. e Vasconcelos, E. (2014). Neurociência e educação: O sistema nervoso e sua relação com a aprendizagem. In: IV Simpósio Nacional de Ensino de Ciência e Tecnologia. Ponta Grossa/PR.

Souza, G. (2016). et al. A neurociência e a educação: como nosso cérebro aprende?. 2016.

Sternbrg, R. (2005) Psicologia Cognitiva. Trad. AM Luche, R Galman. Cengage Learning, São Paulo. 